\title{
Empleo de recursos audiovisuales para dinamizar los procesos de aprendizaje en estudiantes universitarios
}

Cristina López-Cózar Navarro a ${ }^{\mathrm{a}}$ Tiziana Priede Bergamini ${ }^{\mathrm{b}}$ y Sonia Benito Hernández

aUniversidad Politénica de Madrid, cristina.lopezcozar@upm.es, bUniversidad Europea de Madrid, tiziana.priede@universidadeuropea.es y CUniversidad Politénica de Madrid, sonia.benito@upm.es.

\section{\$EWWFW}

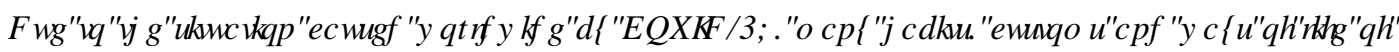

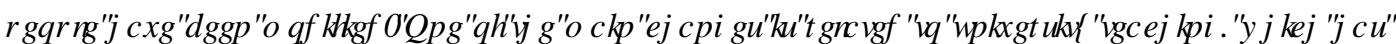

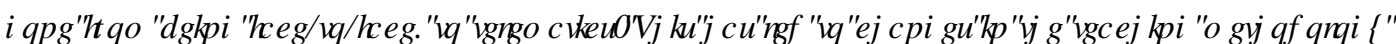

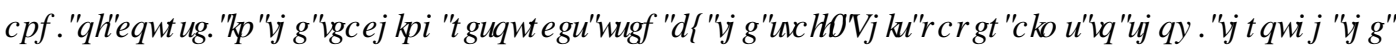

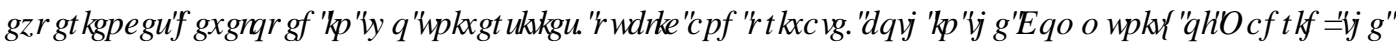

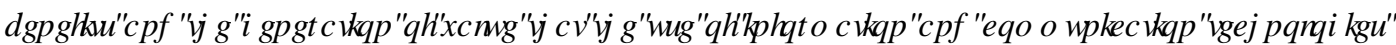

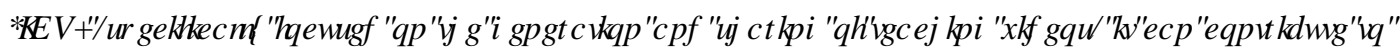

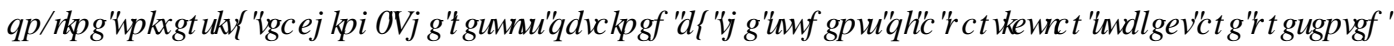

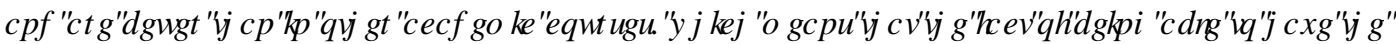

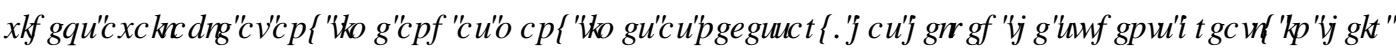
ODLQDISLRFHM

(]

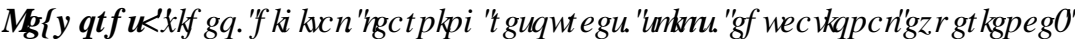

[

\section{HXP HQ}

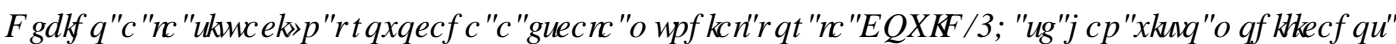

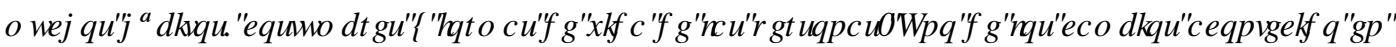

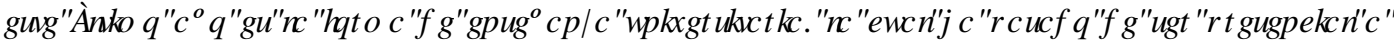

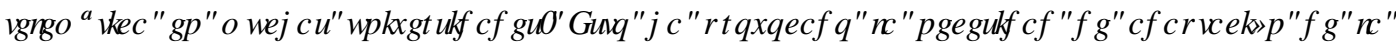

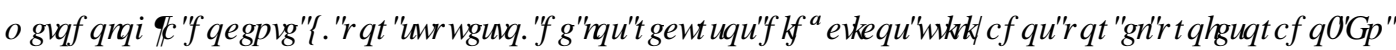

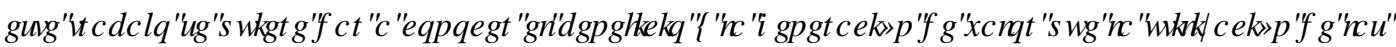

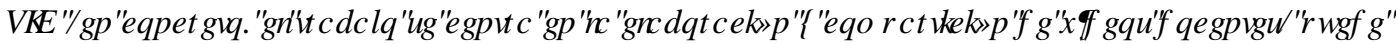

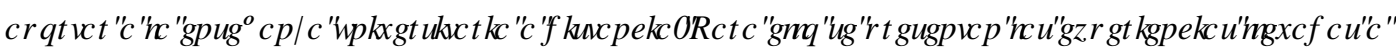

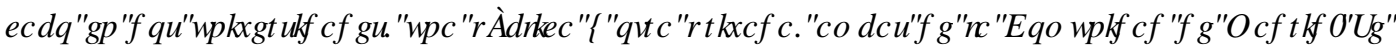

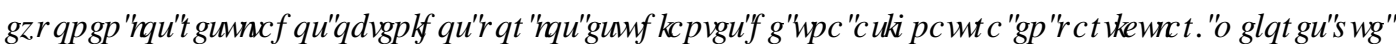

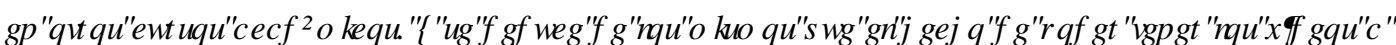

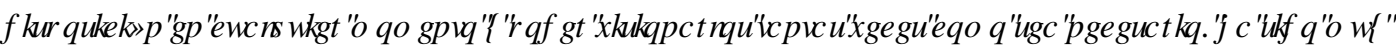
SRUWWRISDWUXISLRFHR]GHDSUHQG]DNFII

]

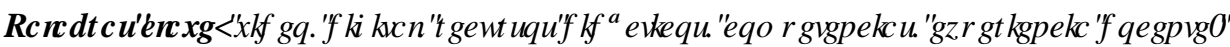




\section{Introducción}

Cuando se cumple un año del inicio de la pandemia provocada por el coronavirus, nos seguimos enfrentando a un entorno altamente incierto y preocupante. La COVID-19 está teniendo un devastador impacto en la población desde el punto de vista de la salud. Así, según datos de la Universidad Johns Hopkins, a finales del mes de febrero de 2021, el número de contagios ya superaba los 113,4 millones de casos en todo el mundo y ha provocado más de 2,5 millones de personas fallecidas. Estas cifras, lamentablemente, siguen creciendo; en estos últimos meses han aparecido nuevas mutaciones del virus de las que aún se desconoce su impacto. Además, y a pesar de que las primeras vacunas ya están disponibles, su distribución y administración está siendo mucho más lenta de lo deseado y, en algunos casos, se analizan y discuten los posibles efectos secundarios de su inoculación.

Como resultado de la rápida expansión del virus, se han producido una serie de cambios en las prácticas sociales y en la forma de vida, tales como los confinamientos domiciliarios y el distanciamiento físico entre las personas. Todas estas medidas están afectando a las relaciones personales, las profesionales y también, sin duda, a las relaciones académicas docente-estudiante. Efectivamente, tanto durante el segundo semestre del curso 2019-20 como, en muchos casos, durante el presente curso, el alumnado no se encontraba en las aulas sino que se conectaba a través de internet y seguía las clases de forma síncrona. Así pues, aunque el soporte tecnológico a la docencia no es nuevo (Aguiar et al., 2019), ahora más que nunca, la utilización de las tecnologías de la información y la comunicación (TIC) como apoyo a la enseñanza se ha convertido en algo impresicindible y habitual en todos los niveles de la educación.

En el caso de la educación superior, la incorporación de las TIC a los programas universitarios lleva mucho tiempo siendo una prioridad en el EEES, y, aunque en la última década ya se ha ido avanzando en este sentido, ha sido en este ultimo año cuando se ha dado un gran avance. No obstante, no en todas las áreas de conocimiento se podrá dar con igual facilidad, pues es cierto que el tema objeto de estudio condiciona los recursos y medios más ajustables y factibles para su impartición.

Siguiendo a Cacheiro González (2011), se puede decir que la aplicación de las TIC a la enseñanza facilita la creación de contenidos, permite la formación a distancia y contribuye a la creación de entornos colaborativos. A su vez, esta misma autora sostiene que las TIC se pueden utilizar para la información, la colaboración y el aprendizaje: los recursos TIC facilitan la obtención de información para complementar y profundizar en las materias; permiten construir un capital social participando en redes de profesionales, con otros docentes, instituciones, etc.; y posibilitan formas diferentes de trabajar para alcazar los resultados de aprendizaje. En resumen, estas tecnologías permiten la ruptura de las barreras espacio-temporales, posibilitan la interacción con la información y se muestran claramente útiles como herramientas de apoyo al aprendizaje (Soto et al., 2009). A pesar de estas ventajas, el empleo de las TIC en las prácticas educativas aún se mantenía por debajo de su potencial hasta hace muy poco tiempo; de hecho, como se ha comentado, su uso se ha visto incrementado en los últimos meses como consecuencia de los efectos de la pandemia.

En educación es posible diseñar modelos híbridos en los que concurren las tres funciones señaladas por Cacheiro González (2011): información, colaboración y aprendizaje. Así, dentro de los recursos TIC, los videos posibilitan crear y compartir contenidos sobre distintas temáticas, ya sean específicas de una asignatura o de carácter más transvesal, realizados por una única persona o como una actividad colaborativa. Además, permiten dotar de flexibilidad y complementar la docencia síncrona, ofreciendo contenidos digitales, específicos y diferenciales que aporten valor añadido a los programas formativos establecidos. En este sentido, si bien el uso de videos didácticos es algo habitual desde hace varios años en los procesos de enseñanza aprendizaje (Camacho Miñano et al., 2016; De la Fuente Sánchez et al., 2018; ArroyoBarrigüete et al., 2019), esta práctica en el momento actual es todavía mayor (Moreno-Guerrero et al., 2020). 


\section{Objetivo}

El objetivo este trabajo es dar a conocer una experiencia docente consistente en la aplicación de herramientas tecnológicas en el proceso de aprendizaje de los estudiantes como apoyo para la adquisición de conocimientos y el desarrollo de competencias. En concreto, se expone la utilización de recursos audiovisuales para fomentar el espíritu emprendedor responsable y sostenible entre el alumando universitario madrileño. Para ello se presentan dos casos reales llevados a cabo en la Universidad Politécnica de Madrid y la Universidad Europea de Madrid, ofrenciendo así ejemplos realizados tanto en una institución pública como en una privada.

Dadas las actuales circunstancias, no ha sido posible llevar a cabo encuentros presenciales entre el alumnado y profesionales externos que promuevan la creación de iniciativas empresariales basadas en el emprendimiento social. De este modo, cada una de las dos universidades ha planteado soluciones basadas en las TIC -concretamente en la grabación y compartición de videos- para poder hacer participar a los emprendedores sociales en el aula, no sólo para compartir sus conocimientos, sino también sus propias vivencias personales.

Consideramos que se trata de una práctica interesante para la comunidad académica, dado que se puede adaptar muy fácilmente para realizarla en diferentes asignaturas relacionadas con el área de administración de empresas de cualquier titulación universitaria y para cualquier tipo de institución. El tema en cuestión objeto del presente estudio, el emprendimiento social, se presta perfectamente para este tipo de actividades; su idoneidad se debe a su relativa novedad y a su carácter social, de vital importancia dadas las circunstancias existentes. Se trata, por tanto, de un tema relevante y de actualidad, donde los vídeos creados y compartidos, con la instantaneidad que caracteriza la sociedad en la que vivimos, ayudan a hacer llegar al estudiante la realidad de lo que implica emprender y de las necesidades sociales que el mismo emprendimiento puede aprovechar y satisfacer.

\section{Desarrollo de la innovación}

La literatura previa pone de manifiesto la utilidad y la aceptación de los videos educativos por parte del alumando (Torres-Ramírez et al., 2014). En particular, diversos trabajos se han desarrollado en el área de administración de empresas, como los llevados a cabo por Agnello et al. (2011), Camacho et al. (2016), De la Fuente Sánchez et al. (2018) y Arroyo-Barrigüete et al. (2019). Así, Camacho et al. (2016) muestran que la mayoría de los estudiantes encuentra los videos prácticos, interesantes y motivadores, y sostienen que el aprendizaje fue mayor al estar realizando actividades relacionadas con proyectos empresariales reales. Por su parte, De la Fuente Sánchez et al. (2018) aportan evidencia empírica que corrobora una relación positiva entre el uso de videos y el rendimiento académico, así como el valor añadido que ofrencen estos recursos en la enseñanza universitaria a distancia.

\subsection{Experiencia en universidad pública}

En el caso de la iniciativa llevada a cabo en la Universidad Politénica de Madrid, la idea de la utilización de material audiovisual surge a partir de la constatación del bajo grado de conocimiento que, en general, tienen los universitarios sobre el emprendimiento social. Con el propósito de averiguar cuánto saben, el interés que suscita y hasta qué punto están dispuestos a involucrarse en proyectos empresariales de este tipo, se realizó una encuesta a un total de trescientos ochenta estudiantes de la Escuela Técnica Superior de Ingeniería Agronómica, Alimentaria y de Biosistemas (ETSIAAB). De las respuestas obtenidas se deduce 
que existe un desconocimiento generalizado sobre el concepto y la utilidad de la empresa social; de hecho, un porcentaje muy bajo de estudiantes afirmaron conocer un caso real de una empresa social. Respecto a los principales canales a partir de los cuales habían oído hablar de este tipo de emprendimiento, la mayoría contestó que fue a través de internet; en segundo lugar, a través de la televisión; un 16 por cierto manifestó que había tenido contacto a partir de otra persona; mientras que charlas o clases en la universidad ocupan el cuarto lugar. La mayoría de los encuestados manifestó su interés por aprender más sobre el tema y casi un 70 por cierto consideraba adecuado que fuera la propia universidad la que se encarguara de dar a conocer el emprendimiento social entre el alumnado.

Los resultados de la encuesta realizada, que como se ha comentado revelan el deseo por tener un mayor conocimiento, muestran la idoneidad de potenciar actividades docentes que favorezcan el acercamiento al emprendimiento social. Sin embargo, se trata de una cuestión compleja, pues en muchas ocasiones no es posible incluir esta temática en las clases, especialmente en aquellas titulaciones que deben priorizar el perfil técnico del alumnado, como son los grados del área de ingeniería. Por ello, se plantearon varias alternativas para estudiar cuál podría ser la forma más apropiada de hacer llegar el emprendimiento social a los jóvenes universtiarios. Finalmente, se consideró que la mejor opción era la creación de contenido audiovisual que pueda ponerse a disposición de un alto número de estudiantes.

Así pues, se ha procedido a la grabación de un video en el que se explica qué es una empresa social y cuáles son sus principales características, así como la importancia de este tipo de iniciativas en el entramado empresarial de cualquier país. En primer lugar, un equipo compuesto por varios docentes y con la colaboración de tres emprendedores sociales preparó un guion con el contenido que debía aparecer. Posteriormente, una alumna diseñó los dibujos, puso voz al video y se ocupó de la parte técnica. Para ello se utilizó el formato Windows Media Video (WMV).

Una vez terminado el proceso de elaboración, se decidió realizar la difusión entre el alumnado universitario a través de la plataforma $0 R R C O$ En este caso, no se trata de un tema incluido en el programa académico de una asignatura en particular, sino relacionado con las competencias trasnversales. De esta manera, el video se ha subido como material complementario al espacio virtual de diversas asignaturas tanto de grado como de postgrado del área de organización de empresas.

\subsection{Experiencia en universidad privada}

En el caso de la iniciativa llevada a cabo en la institución privada Universidad Europea de Madrid, se planteó para la asignatura de Economía de la Empresa de primer curso de los estudiantes del grado en Gestión Deportiva. En dicha asigntura, un 20\% de la calificación se corresponde con la elaboración y presentación de un trabajo basado en el desarrollo de un modelo de negocio de emprendimiento social en el sector deportivo. Concretamente, el proyecto se titula «Innovación social en el deporte». Esta actividad tradicionalmente se ha llevado a cabo con la colaboración de un emprendedor social: el fundador de la plataforma «Mysocialfit». Se trata de una interesante propuesta de negocio que consiste en ofrecer distintas actividades físicas en los parques públicos o espacios abiertos de diversas ciudades, de forma cómoda y flexible para el usuario, a través de una plataforma tecnológica; permite, además, a los profesionales del ejercicio físico encontrar una forma de obtener ingresos por sus servicios y desarrollarse profesionalmente. Dicha plataforma entraría dentro de lo que hoy conocemos como economía colaborativa, en virtud de la cual se permite el intercambio de bienes y servicios basado en las posibilidades que ofrecen los avances tecnológicos. Son nuevos modelos empresariales en los que las actividades se prestan a través de plataformas tecnológicas en las que confluyen los proveedores de servicios, los usuarios de dichos servicios 
y los intermediarios, y cuya función es la de conectar y facilitar las transacciones entre ellos (Comisión Europea [COM], 2016; López-Cózar y Priede, 2021).

En años anteriores a los afectados por la pandemia, este emprendedor acudía al aula a ofrecer una clase o «PDUAFDW sobre emprendimiento social, a explicar su experiencia y a presentar las principales herramientas a disposición de los emprendedores para facilitar el desarrollo de una empresa social. Dadas las dificultades de presencia física y desplazamiento que se han presentado en el curso académico 20202021, se tomó la decisión de preparar un bloque digital para apoyar la colaboración del emprendedor y el mejor desarrollo del proyecto para los estudiantes.

El bloque digital se ha basado en la grabación de 6 vídeos. El primero se centró en una breve entrevista realizada al emprendedor por parte de la profesora de la asigntura en la que se le preguntaba por aspectos generales del emprendimiento social, de las organizaciones del cuarto sector y de la descripción concreta de la plataforma «Mysocialfit». Con este primer vídeo se pretendía contextualizar la actividad y acercar al alumando a la innovación social, además de presentar al ponente y su modelo de negocio. Los siguientes vídeos se centraron en la breve explicación -no más de quice minutos- por parte del ponente de las herramientas más empleadas de emprendimiento: el popular lienzo canvas, el mapa de empatía, el modelo conocido como «EXI HUSHURQD», el canvas de la propuesta de valor y el mínimo producto viable y mínimo producto espectacular. En cada vídeo se explica, de forma clara y concisa, cada herramienta y se ofrece al estudiante un ejemplo para facilitar su comprensión y su puesta en práctica.

El proyecto final de la asigntura, al que se asocia el bloque digital, es un trabajo en equipo que tiene como objetivo la aplicación de los conceptos adquiridos a lo largo del curso, a través del desarrollo de un modelo de negocio y la aplicación de las herramientas de emprendimiento descritas en los vídeos. Los estudiantes deben presentar el proyecto en un plazo establecido; dicho proyecto es valorado tanto por la profesora como por el ponente externo, quien aporta su evaluación y comentarios, los cuales vienen a complementar la evaluación académica de la docente.

\section{Resultados}

En el caso de la Universidad Politénica de Madrid, la temática del emprendimiento social no está asociada a un ámbito disciplinario específico, sino que se incluye como material complementario de diversas asignaturas relacionadas con el área de organización de empresas. Sin embargo, en la actividad llevada a cabo en la Universidad Europea, al realizarse en el contexto de una asignatura concreta, es posible apreciar de manera directa los resultados obtenidos.

En particular, la asignatura Economía de la Empresa se ha impartido en dos grupos durante el primer semestre del curso 2020-2021, y se han presentado un total de nueve proyectos de emprendimiento. Los temas tratados en dichos proyectos han sido de diversa índole, demostrando el alumnado claramente haber comprendido el concepto de la innovación social en el deporte. Así, por ejemplo, entre los objetivos abordados figuran la creación de una entidad deportiva que favorezca la inclusión social de personas con diversas discapacidades intelectuales o la ayuda a determinados colectivos sociales -como personas con obesidad- a realizar entrenamientos RQQQQHguiados por personal especializado.

En cuanto a las calificaciones, las notas han oscilado entre los seis y los nueve puntos, lo cual demuestra que el alumnado ha aprovechado bien la actividad y ha desarrollado a través de la misma los resultados de aprendizaje y las principales competencias de la asigntura. Dado que ninguno de los equipos obtuvo una calificación inferior a cinco puntos, podemos afirmar que esta actividad ha permitido alcanzar dichos objetivos de aprendizaje.

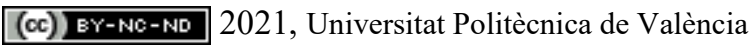

CRQJ UHRT, Q5 HGHपिए। 
Por su parte, estos buenos resultados obtenidos y, más concretamente, las calificaciones alcanzadas en las presentaciones de las herramientas de emprendimiento por parte de los estudiantes, ponen de manifiesto la alta calidad en la realización de las mismas en comparación con los resultados obtenidos en años anteriores. Se observa, por tanto, una relación positiva la utilización de recursos audiovisuales y el rendimiento académico, coincidiendo con la literatura previa (De la Fuente Sánchez et al., 2018). La grabación del bloque digital ha demostrado ser la causa de dicha mejora. En efecto, el tener los vídeos a disposición en cualquier momento y poder visionarlos tantas veces como fuera necesario, ha ayudado mucho a los estudiantes en su proceso de aprendizaje, en línea con los planteamientos de Soto et al. (2009), Cacheiro González (2011) y Camacho et al. (2016).

\section{Conclusiones}

La aplicación de las TIC a la enseñanza universitaria facilita la creación de contenidos, permite la formación a distancia y contribuye a la creación de entornos colaborativos, dinámicos y motivantes para los estudiantes. Esto favorece claramente el proceso de aprendizaje, si bien, hay que tener en cuenta el área de conocimiento y la temática en cuestión, ya que no todos los recursos procedentes de las TIC se adaptan y favorecen este proceso en todas las áreas por igual. Por este motivo, es importante analizar qué recurso es más idóneo para cada tema y área de conocimiento.

En este trabajo se presentan dos ejemplos de la utilización de videos como apoyo del proceso de enseñanzaaprendizaje tanto en la universidad pública como en la privada. Se ha podido comprobar, gracias a los resultados obtenidos por los estudiantes, que el hecho de crear, incluir y comparir vídeos en la docencia telemática ha favorecido su aprendizaje, en comparación con resultados de años antereiores, además de haberles motivado a mejorar su conocimiento del emprendimiento social.

El hecho de tener recursos interactivos, disponibles en todo momento, ha sido clave para mejorar los resultados del trabajo de los estudiantes. Los vídeos han ayudado a la profundización del tema, así como a aclarar las dudas que les han ido surgiendo a lo largo de su proceso de aprendizaje. Su dinamicidad además hace que el esfuerzo del estudiante en el uso de este recurso sea menor que en el uso de otros alternativos por lo que motiva y hace más efectivo su utilización. Sin duda, es una opción muy recomendable.

Especialmente en un entorno como el actual, en el que estamos viviendo un periodo de recuperación tras la pandemia provocada por la COVID-19, muchas de las prácticas tecnológicas que como docentes hemos adoptado para responder a las circunstancias, previsiblemente las incorporaremos en nuestro trabajo diario. Así pues, se aconseja la utilización de recursos procedentes de las TIC, en especial de vídeos, tanto en la docencia a distancia como presencial que apoyen las clases, previa reflexión o análisis sobre la idoneidad de los mismos a la temática o área de conocimiento en cuestión. Por ello, la competencia digital del profesorado, entendida como su aptitud para el empleo de tecnologías digitales en la docencia, se convierte en capacidad imprescindible para afrontar los retos planteados por dicho entorno. 


\section{Referencias}

AGUIAR, B. O., VELÁZQUEZ, R. M., y AGUIAR, J. L. (2019). "Innovación docente y empleo de las TIC en la educación superior”. 5 HLWD( ISDFIRV, Q (2). 8-20. https://www.revistaespacios.com/a19v40n02/a19v40n02p08.pdf.

AGNello, V., PIKAS, B., AGNELlO, A. J., y PIKAS, A. (2011). "Today's learner, preferences in teaching techniques". \$P HJFDQ-RXUDORID\%UQHML( GXFDMRQW (2), 1-9. doi: https://doi.org/10.19030/ajbe.v4i2.3556.

ARROYO-BARRIGÜETE, J. L., LOPEZ-SANCHEZ, J. I., MINGUELA-RATA, B. y RODRIGUEZ-DUARTE, A. (2019). "Use patterns of educational videos: A quantitative study among university students". En: : RUNQJ[ $3 D S H W R Q$ 2 SHDURQVO DQDJ H HQMTI(2), 1-19. Valencia. doi: http://dx.doi.org/10.4995/wpom.v10i2.12625.

CACHEIRO GONZÁLEZ, M. L. (2011 ). "Recursos educativos tic de información, colaboración y aprendizaje”. 3 L [HD

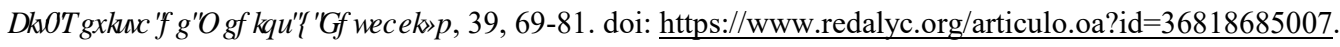

CAMACHO MIÑANO, M. M., URQUÍA GRANDE, E., PASCUAL EZAMA, D. P., y RIVERO MENÉNDEZ, M. J. (2016). "Recursos multimedia para el aprendizaje de Contabilidad Financiera en los grados bilingües". ( GXFDFyQ ; ; ૧, प्(1), 63-89. doi: http://dx.doi.org/10.5944/educXX1.13941.

COMISIÓN EUROPEA (2016). Communication from the Commission to the European Parliament, the Council, the European Economic and Social Committee and the Committee of the Regions - A European Agenda for the Collaborative Economy. COM (2016) 356 final. Brussels. https://ec.europa.eu/transparency/regdoc/rep/1/2016/EN/12016-356-EN-F1-1.PDF

DE LA FUENTE SÁNCHEZ, D., SOLÍS, M. H. y MARTOS, I. P. (2018). "Vídeo educativo y rendimiento académico en la enseñanza superior a distancia”. 5HLWD, EHRDP HUFDQD GH ( GXFDFYQ D ' LWDQFL, पP(1), 323-341. doi: http://dx.doi.org/10.5944/ried.21.1.18326.

LÓPEZ-CÓZAR-NAVARRO, C. y PRIEDE-BERGAMINI, T. (2021). Social Entrepreneurship and Related Concepts: The Path of Opportunity to Foster New Ventures. In Handbook of Research on Nascent Entrepreneurship and Creating New Ventures. 273-294. IGI Global. doi: https://doi.org/10.4018/978-1-7998-4826-4.ch013.

MORENO-GUERRERO, A. J., RODRÍGUEZ-JIMÉNEZ, C., GÓMEZ-GARCÍA, G., y RAMOS NAVAS-PAREJO, M. (2020). "Educational innovation in higher education: Use of role playing and educational video in future teachers' training”. 6XWWQDEICW, प0(6), 2558-2572. doi: http://dx.doi.org/10.3390/su12062558.

SOTO, C., SENRA, A. I., y NEIRA, M. C. (2009). "Ventajas del uso de las TICs en el proceso de enseñanzaaprendizaje desde la óptica de los docentes universitarios españoles". ( ' 8 7( \& 5 HLWD( OFWy QFDDGH7HFRQR tDD ( GXFDMWD, (29), 1-12. doi: https://doi.org/10.21556/edutec.2009.29.451.

TORRES-RAMÍREZ, M., GARCÍA-DOMINGO, B., AGUILERA, J., y DE LA CASA, J. (2014). "Video-sharing educational tool applied to the teaching in renewable energy subjects". \&RPSXWLW I ( GXFDMRQ PQ, 160-177. http://dx.doi.org/10.1016/i.compedu.2013.12.014.

(cc) BY-NC-ND 2021, Universitat Politècnica de València

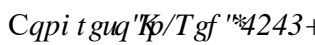

